\title{
Itokawa: The power of ground-based mid-infrared observations
}

\author{
Thomas G. Müller ${ }^{1}$, T. Sekiguchi ${ }^{2}$, M. Kaasalainen ${ }^{3}$, M. Abe ${ }^{4}$ \\ and S. Hasegawa ${ }^{4}$ \\ ${ }^{1}$ Max-Planck-Institut für extraterrestrische Physik, \\ Giessenbachstraße, 85748 Garching, Germany; \\ tmueller@mpe.mpg.de \\ ${ }^{2}$ National Astronomical Observatory of Japan, 2-21-1 Osawa, Mitaka, Tokyo 181-8588, Japan; \\ t.sekiguchi@nao.ac.jp \\ ${ }^{3}$ Department of mathematics and statistics, Gustaf Hallstromin katu 2b, P.O. Box 68, \\ FIN-00014 University of Helsinki, Finland; \\ mjk@rni.helsinki.fi \\ ${ }^{4}$ Institute of Space and Astronautical Science, Japan Aerospace Exploration Agency, 3-1-1 \\ Yoshinodai, Sagamihara, Kanagawa 229-8510, Japan; \\ abe@planeta.sci.isas.jaxa.jp; hasehase@isas.jaxa.jp
}

\begin{abstract}
Pre-encounter ground-based thermal observations of NEA 25143 Itokawa at $10 \mu \mathrm{m}$ led to a size prediction of $520( \pm 50) \times 270( \pm 30) \times 230( \pm 20) \mathrm{m}$, corresponding to an effective diameter of $\mathrm{D}_{\text {eff }}^{T P M}=318 \mathrm{~m}$ (Müller et al. 2005). This is in almost perfect agreement with the final in-situ results $535 \times 294 \times 209 \mathrm{~m}\left(\mathrm{D}_{\text {eff }}^{\text {Hayabusa }}=320 \mathrm{~m}\right.$; Demura et al. 2006$)$. The corresponding radar value, based on the same shape model (Kaasalainen et al. 2005), were about $20 \%$ too high: $594 \times 320 \times 288 \mathrm{~m}\left(\mathrm{D}_{\text {eff }}^{\text {Radar }}=379 \mathrm{~m}\right.$; Ostro et al. 2005$)$. The very simple N-band observations revealed a surface which is dominated by bare rocks rather than a thick regolith layer. This prediction was nicely confirmed by the Hayabusa mission (e.g., Fujiwara et al. 2006; Saito et al. 2006). The ground-based measurements covered three different phase angles which enabled us to determine the thermal properties with unprecedented accuracy and in excellent agreement with the results from the touch-down measurements (Okada et al. 2006; Yano et al. 2006). These thermal values are also key ingredients for high precision Yarkovsky and YORP calculations (mainly the rotation slowing) for Itokawa (e.g., Vokrouhlický et al. 2004; Vokrouhlický et al. 2005). In addition to the above mentioned properties, our data allowed us to derive the surface albedo and to estimate the total mass. We believe that with our well-tested and calibrated radiometric techniques (Lagerros 1996, 1997, 1998; Müller \& Lagerros 1998, 2002; Müller 2002) we have tools at hand to distinguish between monolithic, regolith-covered and rubble pile nearEarth objects by only using remote thermal observations. This project also emphasizes the high and so far not yet fully exploited potential of thermophysical modeling techniques for the NEA/NEO exploration.
\end{abstract}

Keywords. asteroid, surface; infrared, thermal; photometry

\section{Introduction}

The Japanese Hayabusa spacecraft made a successful rendezvous with the target asteroid (25143) Itokawa in late 2005. It investigated the properties of this Apollo-type near-Earth asteroid during several months and performed two touch-downs to collect surface samples. The return of the samples to Earth is currently foreseen for 2010.

In preparation of this interplanetary mission, we performed ground-based thermal observations at around $10 \mu \mathrm{m}$ during the 2001 and 2004 oppositions to study the thermophysical behavior and to determine size, albedo and surface properties of this small 
asteroid. The goal of our work was to support the Hayabusa mission with information on physical and thermal properties of Itokawa well before the encounter and touch-down phases. As a side effect, we wanted to validate and, if necessary, improve our modeling techniques to maximize the outcome of future ground-based mid-IR observing programs to study other potentially hazardous asteroids.

Müller et al. (2005) listed all ground-based mid-IR observations of 25143 Itokawa, including data points by Delbó (2004) and by Sekiguchi et al. (2003), and describe the data reduction and calibration in detail. Here, we briefly present our thermophysical model together with the Itokawa input parameters (Sec. 2) which we used to interpret our observations. The results of our modeling efforts are then discussed in the light of the Hayabusa findings (Sec. 3).

\section{Thermophysical Model (TPM) and input parameters}
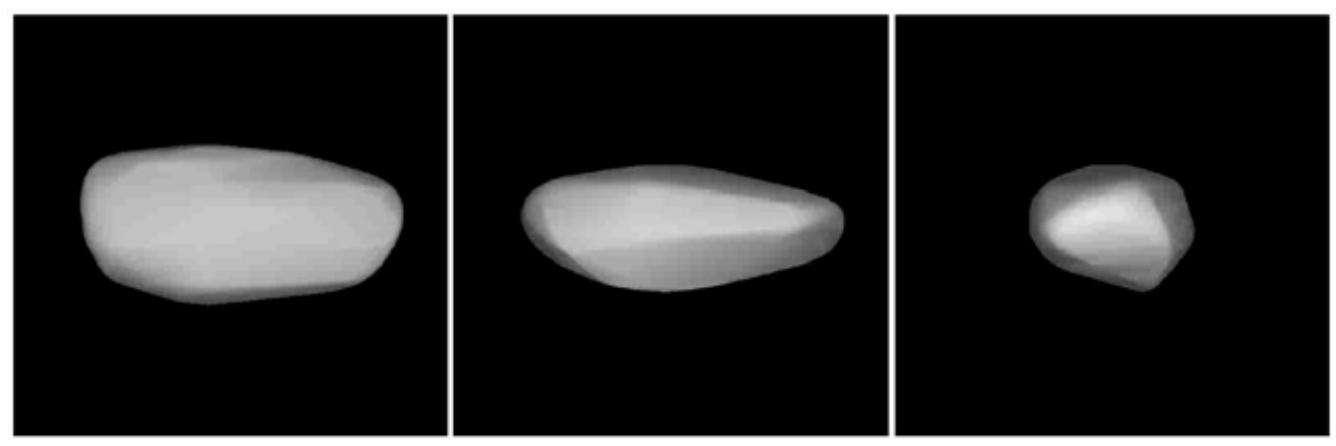

Figure 1. Shape model of 25143 Itokawa through light-curve inversion technique (Kaasalainen et al. 2003).

We applied the TPM by Lagerros $(1996,1997,1998)$ to investigate the physical and thermal properties of the asteroid 25143 Itokawa. On the large scale, the TPM considers the asteroid size, the global shape and spin vector and the actual observing and illumination geometry at the time of an observation. On the small micrometer scale, the TPM takes into account the reflected, absorbed and emitted energy, and also the heat conduction into the surface regolith. The albedo and emissivity control the energy balance and thereby the surface temperature. The thermal inertia in combination with the rotation period and the orientation of the spin vector influence the diurnal temperature variations. As a result, the thermal inertia is strongly connected to the interpretation of mid-IR observations, namely when comparing before and after opposition observations at large phase angles with very different temperatures of the terminator. Moreover, the thermal inertia determines the amplitude of the thermal light-curve for a given aspect angle. The beaming model, described by $\rho$, the r.m.s. of the surface slopes and $f$, the fraction of the surface covered by craters, accounts for the non-isotropic heat radiation, noticeable at phase angles close to opposition. The parameters are dimensionless and they could describe a surface with large craters as well as a rough surface due to bolders of different sizes in combination with micro-impact structures. The beaming model mainly influences the shape of the spectral energy distribution in the mid-IR. But our data are all taken in N-band and slope effects in the spectral energy distribution are not relevant. Our standard set of beaming parameters was therefore the most obvious solution. Various TPM applications for NEAs and main-belt asteroids are described in 
e.g., Müller \& Lagerros (1998, 2002), Müller \& Blommaert (2004), Müller et al. (2004) or Müller et al. (2005).

The following input parameters have been used for the modeling: (i) The H (19.9 mag) and $\mathrm{G}(0.21)$ values describe the absolute visual brightness and the brightness change with phase angle; (ii) We assume a constant emissivity of 0.9 at all mid-IR wavelengths; (iii) We applied the pre-encounter shape-model by Kaasalainen et al. (2005), which is based on large datasets of photometric observations (Fig. 1); the pole solution from lightcurve inversion techniques was $\beta_{\text {pole }}=-89^{\circ} ; \lambda_{\text {pole }}=331^{\circ} ; P_{\text {rot }}=12.13237$ hours; $T_{0}=$ 2451933.95456 and $\phi_{0}=0.0$ (zero-points in time and phase); (iv) We assumed "default" surface roughness parameters (Müller 2002), with $\rho=0.7$ (r.m.s. of the surface slopes) and $\mathrm{f}=0.6$ (fraction of the surface covered by craters).
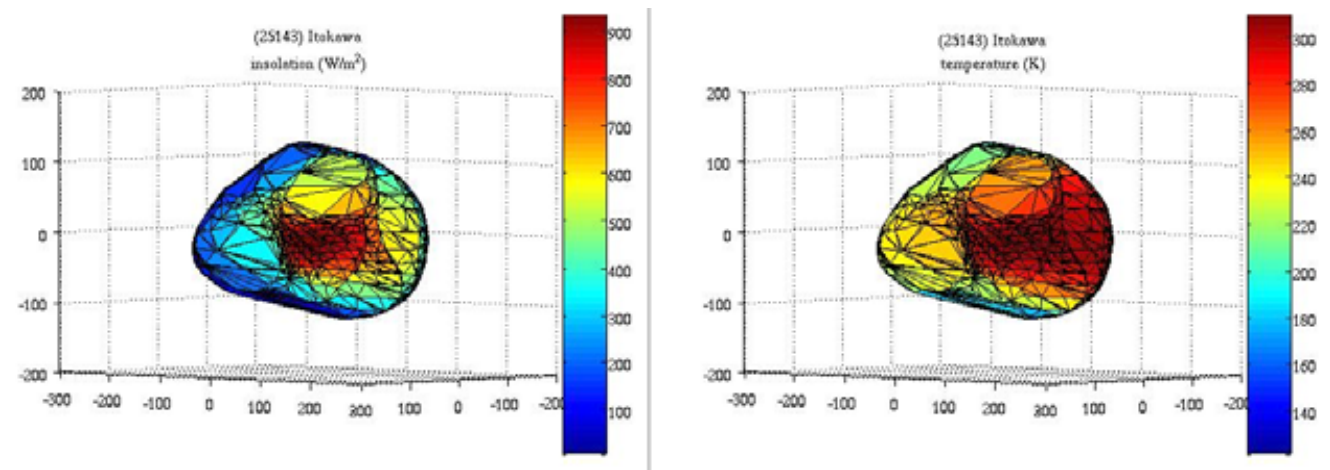

Figure 2. Left: Solar insolation (as seen from the Sun) in $\left[\mathrm{W} / \mathrm{m}^{2}\right]$; Right: Surface temperature (as seen from the Sun) in [K]. The temperature distribution reflects one specific TPM solution for a given set of thermal parameters in combination with the shape model by Kaasalainen et al. (2005): thermal inertia $\Gamma=750 \mathrm{~J} \mathrm{~m}^{-2} \mathrm{~s}^{-0.5} \mathrm{~K}^{-1}$, "standard" beaming model, emissivity $\epsilon=0.9$. The $\mathrm{x}-, \mathrm{y}$ - and z-axes are given in metres, the rotation axis is in z-direction (vertical).

Based on these input parameters and the above mentioned thermal observations, we calculated the effective diameters and albedo values for a range of thermal inertias. The effective diameter is the diameter corresponding to the equal volume sphere and represents the absolute scale factor for the Kaasalainen-shape model. If the shape and thermal models are correct, the effective diameter and albedo values should be independent of the observed wavelength, rotational phase, phase angle or aspect angle (Müller 2002). Müller et al. (2005) demonstrated for 25143 Itokawa the variations of the diameter and albedo solutions as a function of these parameters. We also investigated how the uncertainties of the various input parameters would influence our results. Fig. 2 shows for a thermal inertia $\Gamma=750 \mathrm{~J} \mathrm{~m}^{-2} \mathrm{~s}^{-0.5} \mathrm{~K}^{-1}$ the temperature distribution on the surface. In the asymmetric temperature distribution one can easily recognize the combined rotation and thermal inertia effect. Observing such distributions under large phase angles constrain the interpretation with respect to size and albedo dramatically: The standard deviation of the 20 calculated diameter (or albedo) values changes more than a factor of two for different thermal inertias. Figure 3 illustrates this effect on basis of the $\sigma_{\text {albedo }} /$ albedo values.

\section{Results and Discussion}

The best fit to all our thermal data resulted in an effective diameter of $D_{\text {eff }}=0.32 \pm$ $0.03 \mathrm{~km}$ and an albedo of $p_{V}=0.19_{-0.03}^{+0.11}$. Taking only the highest quality photometric 

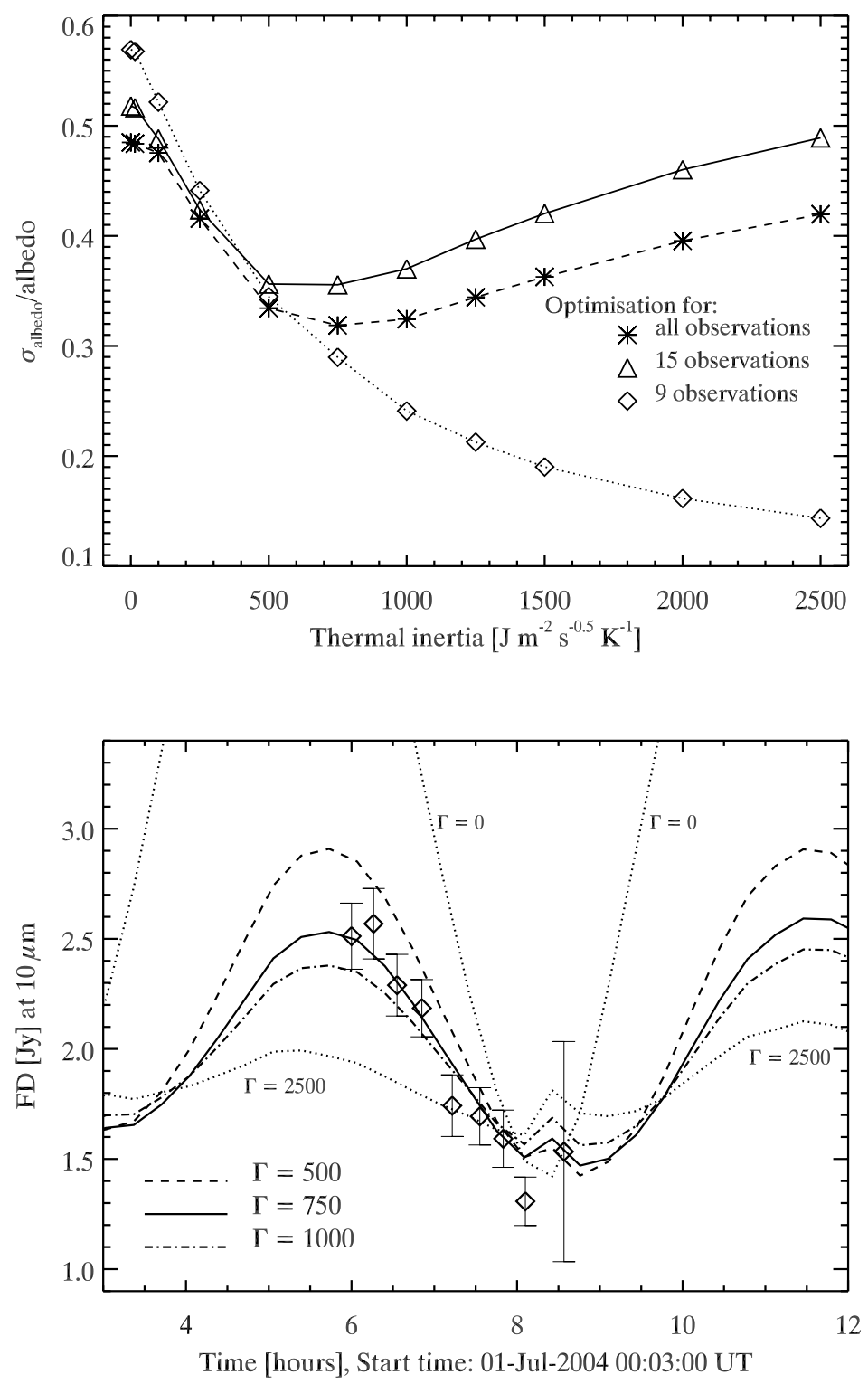

Figure 3. Top: Thermal inertia optimization for the individual TPM albedos and their standard deviation, using 20/15/9 individual observations (dashed/solid/dotted lines). Using only observations from a very small phase angle range (dotted line) does not provide a solution in this optimisation process. Bottom: Predicted thermal light-curve at $10.0 \mu \mathrm{m}$. The original measurements were transported to the $10.0 \mu \mathrm{m}$ wavelength via the TPM. Predictions and measurements are shown with their absolute values. Note that a metallic surface (very high $\Gamma$-values) would produce a very small lightcurve amplitude, while a thick regolith on the surface would increase the amplitude significantly.

data would reduce the size uncertainty to only $\pm 0.01 \mathrm{~km}$, i.e., $10 \mathrm{~m}$ ! The transfer to the slightly asymmetrical and flattened ellipsoid input shape-model gives an absolute size of $520( \pm 50) \times 270( \pm 30) \times 230( \pm 20) \mathrm{m}$. Our size prediction was within $2 \%$ of the final 
Hayabusa results $\left(535 \times 294 \times 209 \mathrm{~m} ; \mathrm{D}_{\text {eff }}^{\text {Hayabusa }}=320 \mathrm{~m}\right.$; Demura et al. 2006). Such an enormous precision was only possible due to the fact that our observations covered a very wide range of phase angles, including measurements at phase angles of $110^{\circ}$ where only a fraction of the surface was illuminated by the sun. Such observing geometries are crucial for thermophysical techniques to see how much a cold or warm terminator contribute to the total measured flux density. It is also interesting to note that the derived size from good quality radar images (Ostro et al. 2005) was overestimated by more than $15 \%$ for unknown reasons. A comparison between our albedo value and the true surface albedo was so far not possible due to the lack of suitable information from the Hayabusa mission.
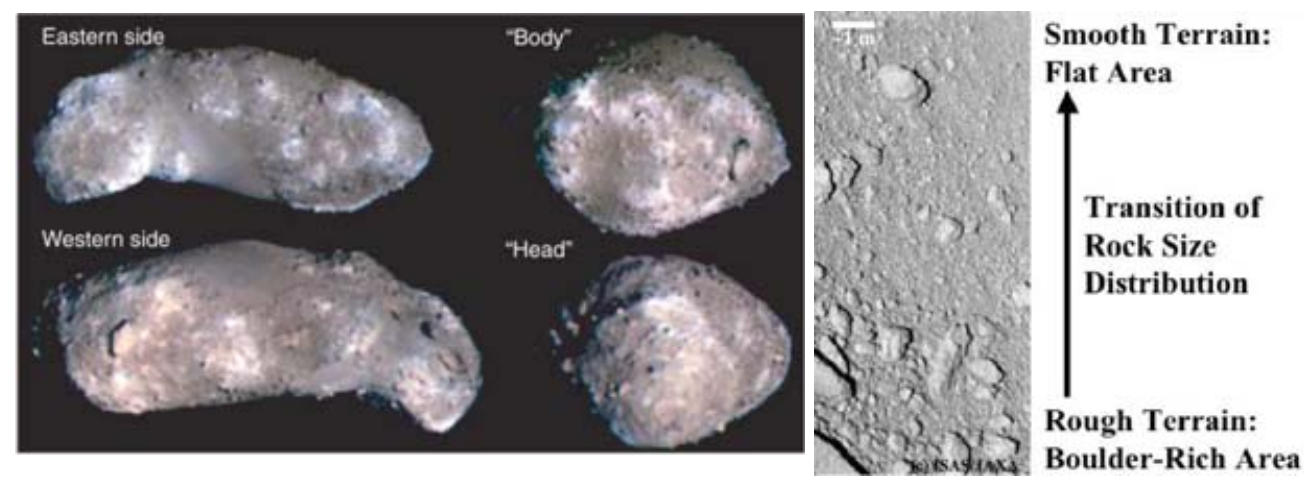

Figure 4. Left: Hayabusa composite color image of Itokawa (Saito et al. 2006). Right: "rocky surface"; image taken from http://www.isas.ac.jp/e/news/2006/0602.shtml

Our final model solution is closely connected to a relatively high thermal inertia of $750 \mathrm{~J} \mathrm{~m}^{-2} \mathrm{~s}^{-0.5} \mathrm{~K}^{-1}$. Only in this way it was possible to find a robust diameter/albedo solution which would fit all observed fluxes (see Fig. 3). A repetition of our optimization calculations with different sub-sets of data confirmed this solution (see solid, dashed and dotted lines in Fig. 3). Robust solutions are only found if the observational data are of similar quality and taken at different phase angles (solid and dashed lines). However, the high thermal inertia was a clear indication for a bare rock dominated surface. A thick dust regolith (typical inertia values of below $50 \mathrm{~J} \mathrm{~m}^{-2} \mathrm{~s}^{-0.5} \mathrm{~K}^{-1}$ ) could be excluded as well as a metallic surface which would have $\Gamma$-values above $10000 \mathrm{~J} \mathrm{~m}^{-2} \mathrm{~s}^{-0.5} \mathrm{~K}^{-1}$ and would produce a very small thermal lightcurve amplitude (see bottom of Fig. 3). This prediction was nicely confirmed by the Hayabusa mission (e.g., Fujiwara et al. 2006; Saito et al. 2006, see also Fig. 4). The ground-based measurements covered three very different phase angles which enabled us to determine the thermal properties with unprecedented accuracy and in excellent agreement with the results from the touch-down measurements (Okada et al. 2006; Yano et al. 2006). These thermal values are also key ingredients for high precision Yarkovsky and YORP calculations (mainly the rotation slowing) for Itokawa (e.g., Vokrouhlický et al. 2004; Vokrouhlický et al. 2005). Based on our modeling and the results from Hayabusa, we also believe that such thermal properties might be an indications for rubble pile structure of a body: monolithic rocks will have very high thermal inertias, while regolith-covered bodies have very low values. We interprete the intermediate range as indicator for an unstable internal structure. The surface of a rubble-pile asteroid will be rearranged by close encounters with large bodies, impacts and vibrational effects. Therefore, the surface conditions are renewed frequently and an Itokawa-like thermal behaviour is the result.

In Fig. 1 the shape model is shown from different orientations; let us compare with two Hayabusa images (Fig. 4). The overall model shape, just based on light-curve inversion 
methods, agrees very well. The same is true for the spin vector. Kaasalainen et al. (2005) derived values of $\beta_{\text {pole }}=-89^{\circ}, \lambda_{\text {pole }}=331^{\circ}$ and $P_{\text {rot }}=12.13237$ hours, in very good agreement with the Hayabusa results of $\beta_{\text {pole }}=-89.66^{\circ}, \lambda_{\text {pole }}=128.5^{\circ}\left(3.9^{\circ}\right.$ error margin; Demura et al. 2006) and $P_{\text {rot }}=12.1324 \pm 0.0001$ hours (Fujiwara et al. 2006).

\section{Conclusions}

Simple observations at thermal mid-IR wavelengths are the key element for the determination of high quality albedo, size and surface properties. Such measurements are easily possible from ground. And, with state-of-the-art instrumentation on large telescopes, even small near-Earth objects can be observed. Shape and spin vector are usually available from visual light-curve inversion techniques. Thermophysical model investigations contribute then the size, albedo and regolith properties. We believe that with our well-tested and calibrated radiometric techniques we have tools at hand to distinguish between monolithic, regolith-covered and rubble pile near-Earth objects by only using remote thermal observations. This project also emphasizes the high and so far not yet fully exploited potential of thermophysical modeling techniques for the NEA/NEO exploration.

\section{Acknowledgements}

We would like to thank F. Hormuth for his support in the data analysis of the TIMMI2 observations and J. Lagerros for his modifications in the TPM code to allow a proper use of the Kaasalainen shape models. M. Delbó supported our re-evaluation of the TIMMI2 data of his thesis work.

\section{References}

Delbó, M. 2004, PhD thesis, FU Berlin, http://www.diss.fu-berlin.de/2004/289/index.html Demura, H., Kobayashi, S., Nemoto, E., et al. 2006, Science 312, 1347

Fujiwara, A., Kawaguchi, J., Uesugi, K., et al. 2006, Science 312, 1330

Kaasalainen, M., Abe, M., Byron, J., et al. 2005, Proceedings of the 1st Hayabusa Symposium, ASP Conf. Series, submitted

Lagerros, J. S. V. 1996, A\&A 310, 1011

Lagerros, J. S. V. 1997, A\&A 325, 1226

Lagerros, J. S. V. 1998, A\&A 332,1123

Müller, T. G. \& Lagerros, J. S. V. 1998, A\& A 338, 340

Müller, T. G. \& Lagerros, J. S. V. 2002, A\& A 381, 324

Müller, T. G. 2002, Meteor. ES Planet. Sci. 37, 1919

Müller, T. G. \& Blommaert, J. A. D. L. 2004, A\& A 418, 347

Müller, T. G., Sterzik, M. F., Schütz, O., Pravec, P., \& Siebenmorgen, R. 2004, A $\mho A$ 424, 1075

Müller, T. G., et al. 2005, A\&A 443, 347

Okada, T., Yamamoto, Y., Inoue, T., et al. 2006, LPS XXXVII, 1965

Ostro, S. J., Benner, L. M., Magri, C., et al. 2005, Meteor. ES Planet. Sci., 40, 1563

Saito, J., Miyamoto, H., Nakamura, R., et al. 2006, Science 312, 1341

Sekiguchi, T., Abe, M., Böhnhardt, H., et al. 2003, A\&\&A 397, 325

Vokrouhlický, D., Čapek, D., Kaasalainen, M. \& Ostro, S. J. 2004, A\&\&A 414, L21

Vokrouhlický, D., Čapek, D., Chesley, S. R. \& Ostro, S. J. 2005, Icarus 173, 166

Yano, H., Kubota, T., Miyamoto, H., et al. 2006, Science 312, 1350 